\title{
Valvectomy versus replacement for the surgical treatment of infective tricuspid valve endocarditis: a systematic review and meta-analysis
}

\author{
Jessica G. Y. Luc ${ }^{1}$, Jae-Hwan Choi ${ }^{2}$, Karishma Kodia ${ }^{2}$, Matthew P. Weber ${ }^{2}$, Dylan P. Horan ${ }^{2}$, \\ Elizabeth J. Maynes ${ }^{2}$, Laura A. Carlson ${ }^{2}$, H. Todd Massey ${ }^{2}$, John W. Entwistle ${ }^{2}$, Rohinton J. Morris ${ }^{2}$, \\ Vakhtang Tchantchaleishvili ${ }^{2}$ \\ ${ }^{1}$ Division of Cardiovascular Surgery, Department of Surgery, University of British Columbia, Vancouver, British Columbia, Canada; ${ }^{2}$ Division of \\ Cardiac Surgery, Thomas Jefferson University, Philadelphia, Pennsylvania, USA \\ Correspondence to: Vakhtang Tchantchaleishvili, MD. Assistant Professor of Surgery, Division of Cardiothoracic Surgery, Thomas Jefferson \\ University, 1025 Walnut St, Suite 607, Philadelphia, PA 19107, USA. Email: Vakhtang.Tchantchaleishvili@jefferson.edu.
}

Background: Optimal surgical treatment of infective tricuspid valve endocarditis in patients with intravenous drug use (IVDU) remains controversial. Tricuspid valvectomy has been proposed for infective tricuspid valve endocarditis in this patient population given the inherent social concerns. The aim of this systematic review and meta-analysis was to compare outcomes of valvectomy versus replacement for the surgical treatment of isolated infective tricuspid valve endocarditis.

Methods: An electronic search was performed to identify all relevant studies published. After assessment for inclusion and exclusion criteria, 16 original studies were pooled for systematic review and meta-analysis.

Results: There were a total of 752 patients with infective tricuspid valve endocarditis, of which $14 \%$ underwent valvectomy and 86\% underwent replacement (mean follow-up 4.2 years, 95\% CI, 1.9-6.4 years). The most common indications for surgical intervention were septic pulmonary embolism in the valvectomy group (74\%, 95\% CI, 28-95\%) and persistent sepsis in the replacement group (62\%, 95\% CI, 31-86\%). There were no differences in rates of stroke [valvectomy 4\% (95\% CI, 1-11\%) vs. replacement 3\% (95\% CI, 1-16\%), $\mathrm{P}=0.85]$ but there was increased likelihood of prolonged ventilation in those who underwent valvectomy [valvectomy 40\% (95\% CI, 30-51\%) vs. replacement 26\% (95\% CI, 23-30\%), P<0.01]. There were no differences in 30-day post-operative mortality [valvectomy 13\% (95\% CI, 5-30\%) vs. replacement $7 \%$ (95\% CI, 5-10\%), P=0.21], post-operative right heart failure [valvectomy $27 \%$ (95\% CI, 10-53\%) vs. replacement $11 \%(95 \% \mathrm{CI}, 5-25 \%), \mathrm{P}=0.17$ ] and recurrent endocarditis [valvectomy $7 \%(95 \% \mathrm{CI}$, $2-23 \%$ ) vs. replacement $19 \%$ (95\% CI, 12-28\%), $\mathrm{P}=0.81$ ]. Valvectomy had a higher rate of tricuspid valve reoperation [valvectomy 56\% (95\% CI, 15-90\%) vs. initial replacement 14\% (95\% CI, 7-27\%), P=0.06].

Conclusions: Tricuspid valvectomy is an acceptable initial therapy for infective tricuspid valve endocarditis in patients with IVDU, providing a bridge to identify those who will self-select as candidates for staged valve replacement.

Keywords: Endocarditis; tricuspid valve; intravenous drug user (IVDU); surgery; valve replacement; valvectomy

Submitted May 26, 2019. Accepted for publication Oct 31, 2019.

doi: $10.21037 /$ acs.2019.11.06

View this article at: http://dx.doi.org/10.21037/acs.2019.11.06 


\section{Introduction}

Infective endocarditis is a disease with substantial morbidity and mortality (1). The current opioid epidemic is associated with increasing rates of intravenous drug use (IVDU) and thereby, an increased incidence of tricuspid valve endocarditis with the number of cases roughly estimated to be 1.5 to 3.3 cases per 1,000 person-years in the United States (2). Medical management for IVDU-associated infective tricuspid valve endocarditis remains the mainstay of therapy, with surgery reserved for cases of infective endocarditis complications, hemodynamic instability, failed medical therapy, persistent embolization or large perivalvular vegetations (3).

Optimal surgical treatment of infective tricuspid valve endocarditis in patients with IVDU remains controversial and includes valve repair or replacement, as well as the removal of the tricuspid valve leaflets and chordae tendinae without replacement (valvectomy) (3). Patients with IVDU infective endocarditis have been reported to have a 10fold higher risk of death or reoperation following surgical therapy compared to those without IVDU [hazard ratio (HR) 9.8, 95\% CI, 2.7-35.3] (1). Although valve repair is the preferred option (3-5), often patients with IVDUassociated tricuspid infective endocarditis present with extensive valvular destruction that precludes valvular repair. Tricuspid valvectomy has been proposed for infective tricuspid valve endocarditis in this patient population given the inherent social concerns with compliance to therapy, abstinence of IVDU and increased perioperative risk with reoperation of infected prosthetic valves (6).

The aim of this systematic review and meta-analysis was to compare outcomes of valvectomy versus replacement for the surgical treatment of isolated infective tricuspid valve endocarditis.

\section{Methods}

\section{Literature search strategy}

Studies comparing tricuspid valvectomy versus valve replacement in patients with IVDU infective tricuspid valve endocarditis were identified through thorough electronic searches performed in September 2018 using Ovid Medline, Embase, Cochrane Central Register of Controlled Trials (CCTR), Cochrane Database of Systematic Reviews (CDSR), Web of Science, Scopus and Cumulative Index of Nursing and Allied Health Literature (CINAHL). To achieve the maximum sensitivity of the search strategy, we combined the terms: "endocarditis", "valvectomy", "valve replacement", "tricuspid", "infective endocarditis" as either key words or MeSH terms. The reference lists of all retrieved articles were reviewed and assessed for further identification of potentially relevant studies using the inclusion and exclusion criteria.

\section{Selection criteria}

Eligibility of studies for the present systematic review and meta-analysis was determined prior to commencement of data collection. These included those in which at least four patients with IVDU underwent either tricuspid valvectomy or valve replacement for the surgical treatment of their isolated tricuspid valve infective endocarditis. Studies including concurrent valvular pathology (non-isolated tricuspid valve infective endocarditis) were excluded. When institutions published duplicate studies with overlapping individual patient data, only the most complete reports were included for quantitative assessment at each time interval. All publications were limited to those involving human subjects and in the English language.

\section{Data extraction}

A quality assessment was performed on all studies included using the Newcastle-Ottawa Scale (NOS), recommended by the Cochrane Manual $(7,8)$ (Table S1). Since all studies had a NOS score 5 or higher, and none were of poor quality, sensitivity analysis was not performed. All data were extracted from article texts, tables, and figures by two investigators independently. Discrepancies between the two investigators were resolved by discussion and consensus. Where data were not available, attempts were made to contact corresponding authors to obtain the relevant data for the current study.

\section{Statistical analysis}

For dichotomous variables, a meta-analysis of proportions with logit transformation was conducted for the available main perioperative and postoperative variables. Continuous data were combined via meta-analysis with random-effects model. Heterogeneity was evaluated using Cochran Q and $\mathrm{I}^{2}$ test. Odds ratio was the utilized effect measure. Subgroup analyses were conducted to compare baseline characteristics and outcomes of patients who underwent tricuspid valvectomy $v s$. replacement. Egger's regression test for funnel plot asymmetry was performed to assess 


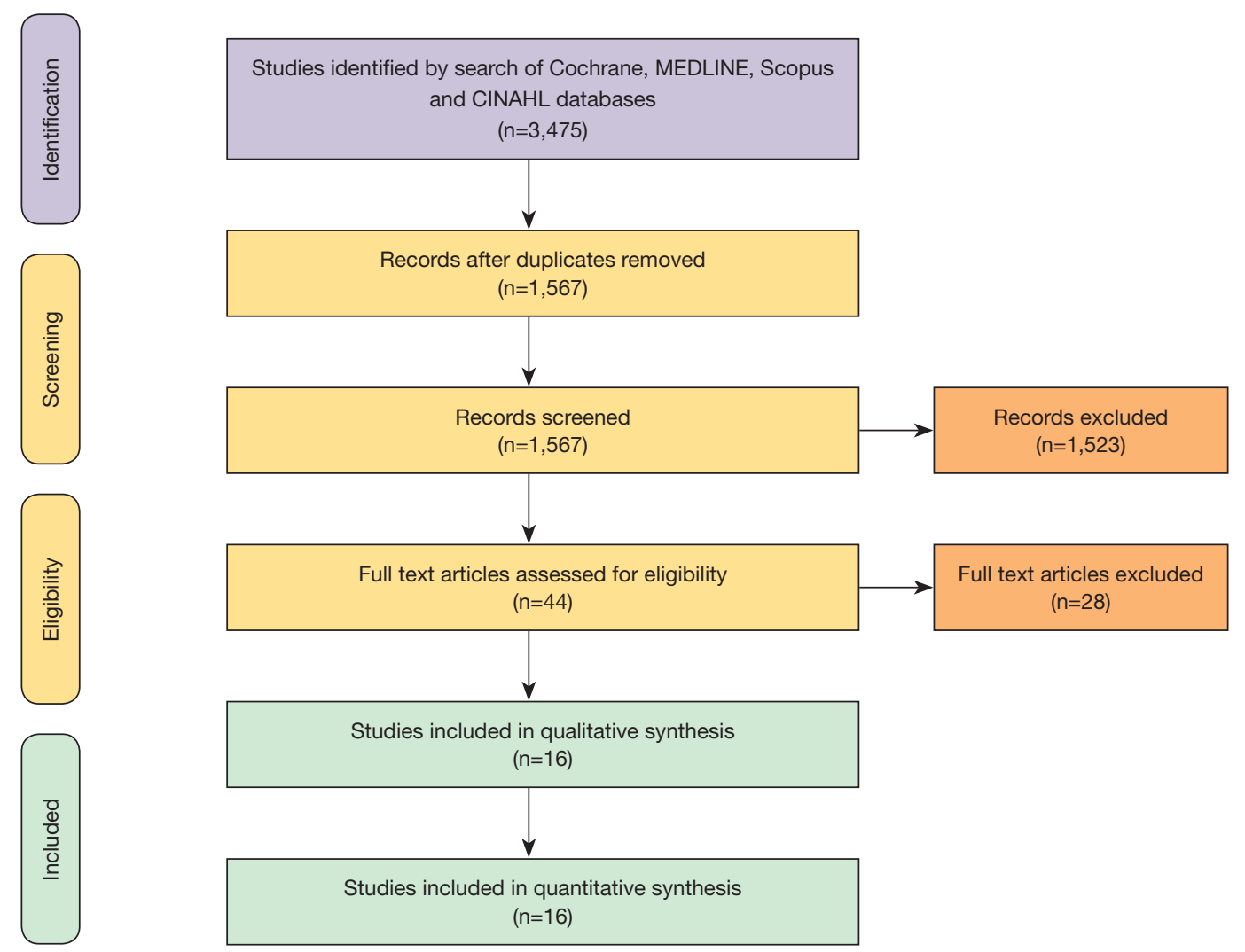

Figure 1 PRISMA schematic of the search strategy. PRISMA, Preferred Reporting Items for Systematic Reviews and Meta-Analyses.

for publication bias. All analyses were performed with $\mathrm{R}$ software, version 3.5.1 (R Foundation for Statistical Computing, Vienna, Austria). P values $<0.05$ were considered statistically significant.

\section{Results}

\section{Study characteristics}

Overall, 1,567 records were identified in the literature search. Following application of the inclusion and exclusion criteria, 16 studies containing 752 patients were included for analysis. Of a total of 752 patients with IVDU infective tricuspid valve endocarditis, $14 \%$ underwent valvectomy and $86 \%$ underwent replacement. A PRISMA flow diagram depicting the overall search strategy is provided in Figure 1. A manual search of references did not yield further studies.

\section{Baseline demographics}

Baseline patient demographics are shown in Table 1. The mean age of patients undergoing surgery (valvectomy or replacement) for tricuspid endocarditis was 36 years old (95\% CI, 26-45 years old), of which $55 \%$ of all patients (95\% CI, 47-62\%) were male. More patients who underwent tricuspid valve replacement had systemic hypertension [valvectomy 20\% (95\% CI, 11-32\%) vs. replacement 35\% (95\% CI, 31-39\%), $\mathrm{P}=0.02$ ].

The most common indications for surgical therapy in patients with IVDU infective endocarditis were persistent sepsis [valvectomy $47 \%$ (95\% CI, 24-71\%) vs. replacement $62 \%$ (95\% CI, 31-86\%), $\mathrm{P}=0.45]$ and septic pulmonary embolism [valvectomy 74\% (95\% CI, 28-95\%) vs. replacement 38\% (95\% CI, 16-66\%), P=0.18].

Patients who underwent tricuspid valvectomy were more likely to have had an infection with Staphylococcus aureus [valvectomy 93\% (95\% CI, 74-98\%) vs. replacement $49 \%$ (95\% CI, 38-61\%), $\mathrm{P}<0.01]$. Whereas, patients who underwent tricuspid valve replacement were more likely to have had an infection with Streptococcus [valvectomy $3 \%$ (95\% CI, 0-21\%) vs. replacement 33\% (95\% CI, 20-50\%), P=0.01], Enterococcus [valvectomy 3\% (95\% CI, 


\begin{tabular}{|c|c|c|c|c|c|c|c|c|c|c|c|c|c|}
\hline \multirow[b]{2}{*}{ Characteristic } & \multicolumn{4}{|c|}{ Tricuspid valvectomy $(n=106)$} & \multicolumn{4}{|c|}{ Tricuspid valve replacement $(n=646)$} & \multicolumn{4}{|c|}{ Overall $(n=752)$} & \multirow[b]{2}{*}{$\begin{array}{l}\mathrm{P} \\
\text { value }\end{array}$} \\
\hline & $\begin{array}{l}\text { Pooled } \\
\text { value } \\
{[95 \% \mathrm{Cl}]}\end{array}$ & $\begin{array}{l}\text { No. of } \\
\text { studies }\end{array}$ & $\begin{array}{l}\text { No. of } \\
\text { patients }\end{array}$ & $\begin{array}{l}1^{2} \\
(\%)\end{array}$ & $\begin{array}{l}\text { Pooled } \\
\text { value } \\
{[95 \% \mathrm{Cl}]}\end{array}$ & $\begin{array}{l}\text { No. of } \\
\text { studies }\end{array}$ & $\begin{array}{l}\text { No. of } \\
\text { patients }\end{array}$ & $\begin{array}{l}1^{2} \\
(\%)\end{array}$ & $\begin{array}{l}\text { Pooled } \\
\text { value } \\
{[95 \% \mathrm{Cl}]}\end{array}$ & $\begin{array}{l}\text { No. of } \\
\text { studies }\end{array}$ & $\begin{array}{l}\text { No. of } \\
\text { patients }\end{array}$ & $\begin{array}{l}1^{2} \\
(\%)\end{array}$ & \\
\hline \multicolumn{14}{|c|}{ Baseline demographics } \\
\hline Male (\%) & $48[38-58]$ & 3 & $47 / 98$ & 0 & $62[50-74]$ & 10 & $308 / 589$ & 45 & 55 [47-62] & 11 & $355 / 687$ & 29 & 0.07 \\
\hline $\begin{array}{l}\text { Systemic } \\
\text { hypertension } \\
(\%)\end{array}$ & 20 [11-32] & 2 & $18 / 91$ & 21 & 35 [31-39] & 2 & $179 / 516$ & 0 & 26 [17-37] & 2 & $197 / 607$ & $66^{*}$ & 0.02 \\
\hline $\begin{array}{l}\text { Septic } \\
\text { pulmonary } \\
\text { embolism (\%) }\end{array}$ & 74 [28-95] & 2 & $26 / 33$ & $78^{*}$ & 38 [16-66] & 7 & $25 / 72$ & $64^{*}$ & 48 [25-72] & 7 & $51 / 105$ & $72^{*}$ & 0.18 \\
\hline \multicolumn{14}{|l|}{ Microorganisms } \\
\hline $\begin{array}{l}\text { Staphylococcus } \\
\text { Aureus (\%) }\end{array}$ & 93 [74-98] & 2 & $30 / 32$ & 0 & 49 [38-61] & 8 & $37 / 73$ & 0 & 66 [48-80] & 9 & $67 / 105$ & 48 & $<0.01$ \\
\hline $\begin{array}{l}\text { Streptococcus } \\
\text { (\%) }\end{array}$ & 3 [0-21] & 2 & $0 / 32$ & 0 & 33 [20-50] & 3 & $12 / 36$ & 0 & 23 [10-46] & 5 & $12 / 68$ & 40 & 0.01 \\
\hline $\begin{array}{l}\text { Enterococcus } \\
(\%)\end{array}$ & $3[0-21]$ & 2 & $0 / 32$ & 0 & 25 [12-45] & 2 & $7 / 28$ & 0 & 15 [4-42] & 4 & $7 / 60$ & 41 & 0.04 \\
\hline
\end{tabular}

0-21\%) vs. replacement 25\% (95\% CI, 12-45\%), $\mathrm{P}=0.04]$ or fungal organisms [valvectomy 7\% (95\% CI, 1-35\%) vs. replacement 60\% (95\% CI, 19-90\%), $\mathrm{P}=0.03$ ].

\section{Operative outcomes}

The overall follow-up time for both groups was 50 months [valvectomy, 21 months (95\% CI, 0-109 months) vs. replacement 53 months (95\% CI, 24-82 months)], with no statistically significant differences in follow-up time between the two groups $(\mathrm{P}=0.50)$.

There were no differences in postoperative rates of major bleeding [valvectomy $8 \%$ (95\% CI, 2-25\%) vs. replacement 9\% (95\% CI, 3-23\%), P=0.83], stroke [valvectomy 4\% (95\% CI, 1-11\%) vs. replacement 3\% (95\% CI, 1-16\%), $\mathrm{P}=0.85$ ] or hospital readmissions [valvectomy $13 \%$ (95\% CI, 0-84\%) vs. replacement 22\% (95\% CI, 13-35\%), $\mathrm{P}=0.72]$.
Patients who underwent valvectomy were more likely to have had prolonged ventilation time postoperatively [valvectomy 40\% (95\% CI, 30-51\%) vs. replacement $26 \%$ (95\% CI, 23-30\%), $\mathrm{P}<0.01$ ] (Table 2).

Postoperative right heart failure was $27 \%$ (95\% CI, $10-53 \%)$ in the valvectomy group vs. $11 \%$ (95\% CI, 5-25\%) in the replacement group $(\mathrm{P}=0.17)$, recurrent endocarditis occurred in $7 \%$ (95\% CI, 2-23\%) of valvectomy vs. $19 \%$ $(95 \% \mathrm{CI}, 12-28 \%)$ in the replacement group $(\mathrm{P}=0.81)$ as shown in Figures 2,3. Perioperative all-cause reoperation was $8 \%(95 \%$ CI, 2-25\%) in the valvectomy vs. $15 \%(95 \%$ CI, $12-18 \%)$ in the replacement group $(\mathrm{P}=0.30)$. There was a trend towards higher rates of delayed reoperation for tricuspid valve replacement in the valvectomy group $56 \%$ (95\% CI, 15-90\%) vs. re-replacement after initial replacement $14 \%(95 \% \mathrm{CI}, 7-27 \% ; \mathrm{P}=0.06)$ (Table 2, Figure 4). Freedom from reoperation at six months was 


\begin{tabular}{|c|c|c|c|c|c|c|c|c|c|c|c|c|c|}
\hline & \multicolumn{4}{|c|}{ Tricuspid valvectomy $(n=106)$} & \multicolumn{4}{|c|}{ Tricuspid valve replacement $(n=646)$} & \multicolumn{4}{|c|}{ Overall $(n=752)$} & \multirow[b]{2}{*}{$\begin{array}{l}\mathrm{P} \\
\text { value }\end{array}$} \\
\hline & $\begin{array}{l}\text { Pooled } \\
\text { value } \\
{[95 \% \mathrm{Cl}]}\end{array}$ & $\begin{array}{l}\text { No. of } \\
\text { studies }\end{array}$ & $\begin{array}{l}\text { No. of } \\
\text { patients }\end{array}$ & $\begin{array}{l}\mathrm{I}^{2} \\
(\%)\end{array}$ & $\begin{array}{l}\text { Pooled } \\
\text { value } \\
{[95 \% \mathrm{Cl}]}\end{array}$ & $\begin{array}{l}\text { No. of } \\
\text { studies }\end{array}$ & $\begin{array}{l}\text { No. of } \\
\text { patients }\end{array}$ & $\begin{array}{l}1^{2} \\
\text { (\%) }\end{array}$ & $\begin{array}{l}\text { Pooled } \\
\text { value } \\
{[95 \% \mathrm{Cl}]}\end{array}$ & $\begin{array}{l}\text { No. of } \\
\text { studies }\end{array}$ & $\begin{array}{l}\text { No. of } \\
\text { patients }\end{array}$ & $\begin{array}{l}1^{2} \\
(\%)\end{array}$ & \\
\hline $\begin{array}{l}\text { Follow-up } \\
\text { (months) }\end{array}$ & $21[0-109]^{\dagger}$ & 1 & 7 & NA & 53 [24-82] & 4 & 59 & 0 & $50[23-77]$ & 5 & 66 & 0 & 0.50 \\
\hline \multicolumn{14}{|l|}{ Complications } \\
\hline $\begin{array}{l}\text { Major } \\
\text { bleeding (\%) }\end{array}$ & 8 [2-25] & 2 & $7 / 91$ & 32 & 9 [3-23] & 3 & $77 / 540$ & 50 & 10 [6-17] & 5 & $84 / 631$ & 43 & 0.83 \\
\hline $\begin{array}{l}\text { Prolonged } \\
\text { ventilation } \\
(\%)\end{array}$ & 40 [30-51] & 3 & $40 / 99$ & 8 & 26 [23-30] & 4 & $137 / 525$ & 0 & 32 [24-41] & 7 & $177 / 624$ & 49 & $<0.01$ \\
\hline $\begin{array}{l}\text { Reoperation } \\
(\%)\end{array}$ & 8 [2-25] & 2 & $7 / 91$ & 32 & 15 [12-18] & 7 & $83 / 577$ & 0 & 14 [10-18] & 9 & $90 / 668$ & 5 & 0.30 \\
\hline Stroke (\%) & $4[1-11]$ & 2 & $3 / 91$ & 0 & $3[1-16]$ & 4 & $7 / 543$ & 63 & $3[1-8]$ & 6 & $10 / 634$ & 51 & 0.85 \\
\hline $\begin{array}{l}\text { Postoperative } \\
\text { RHF, all (\%) }\end{array}$ & 27 [10-53] & 2 & $4 / 15$ & $0^{*}$ & 11 [5-25] & 6 & $3 / 44$ & 0 & 16 [9-29] & 8 & $7 / 59$ & 0 & 0.17 \\
\hline $\begin{array}{l}\text { Recurrent } \\
\text { endocarditis, } \\
\text { all (\%) }\end{array}$ & 7 [2-23] & 3 & $1 / 40$ & 0 & 19 [12-28] & 11 & $16 / 102$ & 0 & 17 [11-25] & 14 & $17 / 142$ & 0 & 0.81 \\
\hline $\begin{array}{l}\text { Readmission, } \\
\text { all (\%) }\end{array}$ & 13 [0-84] & 2 & $3 / 32$ & $80^{*}$ & 22 [13-35] & 3 & $12 / 54$ & 0 & 23 [13-37] & 3 & $15 / 86$ & 22 & 0.72 \\
\hline $\begin{array}{l}\text { Reoperation } \\
\text { with tricuspid } \\
\text { valve } \\
\text { replacement, } \\
\text { all (\%) }\end{array}$ & 56 [15-90] & 3 & $23 / 40$ & 70 & 14 [7-27] & 10 & $4 / 51$ & 0 & 24 [12-43] & 12 & $27 / 91$ & $47^{\star}$ & 0.06 \\
\hline $\begin{array}{l}\text { Mortality, } 30 \\
\text { days (\%) }\end{array}$ & 13 [5-30] & 4 & $12 / 106$ & 46 & 7 [5-10] & 12 & $37 / 609$ & 0 & 9 [7-13] & 16 & $49 / 715$ & 11 & 0.21 \\
\hline
\end{tabular}

comparable (valvectomy $97 \%$ vs. replacement $89 \%$, $\mathrm{P}=0.30$ ), and at one year trended toward being lower in the valvectomy group (valvectomy $46 \%$ vs. replacement $89 \%$; $\mathrm{P}=0.06$ ) (Table 3).

\section{Mortality and survival}

Postoperative 30-day mortality was 13\% (95\% CI, 5-30\%) in the valvectomy group, and 7\% (95\% CI, 5-10\%) in the replacement group, without statistical significance $(\mathrm{P}=0.21)$ (Table 2, Figure 5). Survival was comparable at six months (valvectomy $77 \%$ vs. replacement $83 \% ; \mathrm{P}=0.69$ ) and one year (valvectomy $77 \%$ vs. replacement $82 \% ; \mathrm{P}=0.70$ ) (Figure 6).

\section{Discussion}

The present systematic review and meta-analysis demonstrates a non-significant trend towards higher postoperative right heart failure and 30-day mortality, and lower recurrent endocarditis and all-cause reoperation in the valvectomy group. Six-month and one-year survival was comparable between the groups. Patients who underwent valvectomy had higher rates of prolonged ventilation and 
A

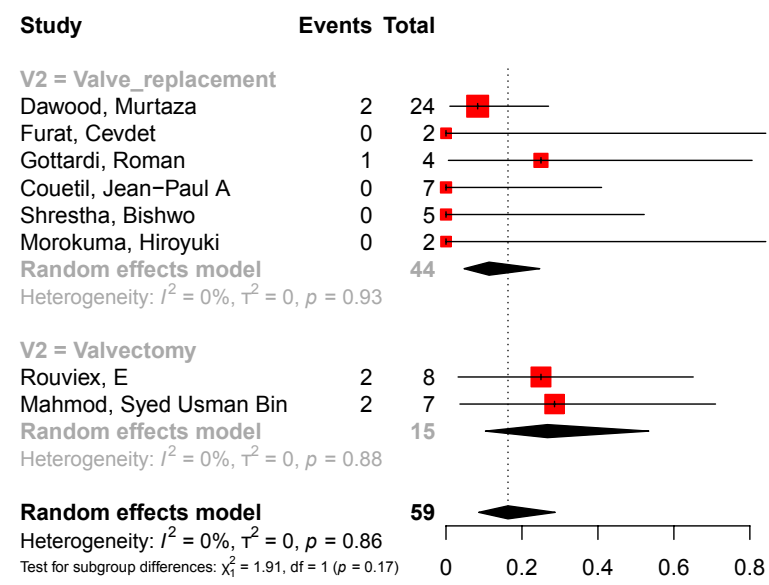

B

Proportion $\quad 95 \%-\mathrm{Cl}$ Weight

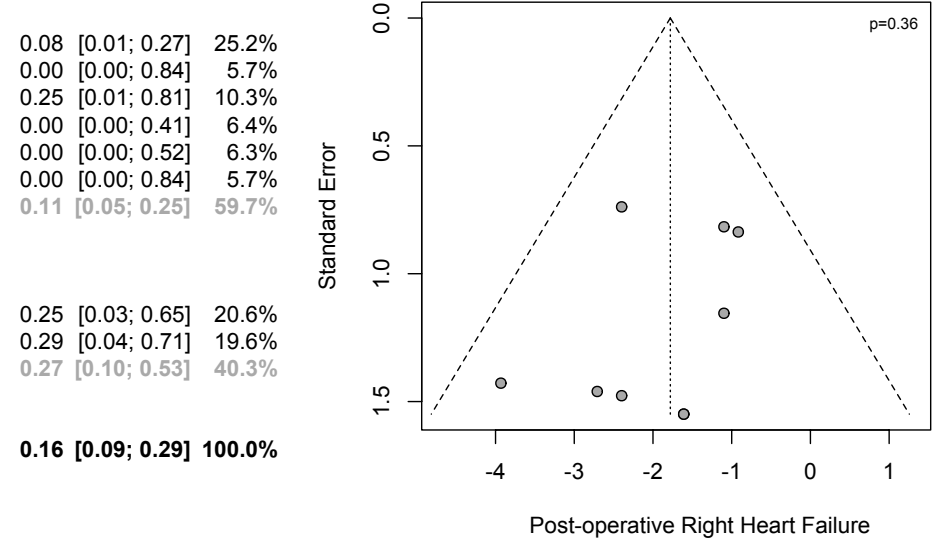

$0.08[0.01 ; 0.27] \quad 25.2 \%$

$0.00[0.00,0.84] \quad 5.7 \%$

$5.7 \%$

$0.11[0.05 ; 0.25]-59.7 \%$

$0.25[0.03 ; 0.65]-20.6 \%$

$0.29[0.04 ; 0.71] \quad 19.6 \%$

$0.27[0.10 ; 0.53] \quad 40.3 \%$

Post-operative Right Heart Failure

Figure 2 Mean postoperative right heart failure rates for patients who underwent valvectomy $v s$. tricuspid valve replacement. (A) Forest plot; (B) funnel plot.

A

$$
\text { Study }
$$

Events Total

$\mathrm{V}_{2}=$ Valve_replacement

Xu, Zhiwei

Dawood, Murtaza

Furat, Cevdet

Pomar, Jose L

Gottardi, Roman

Shrestha, Bishwo

Shetty, Nabha

Jiang, Sheng-li

Morokuma, Hiroyuki

Protos, Adam

Mahmod, Syed Usman Bin

Random effects model

Heterogeneity: $I^{2}=0 \%, T^{2}=0, p=0.91$

$\mathrm{V} 2$ = Valvectomy

Rouviex, E

Protos, Adam

Mahmod, Syed Usman Bin

Random effects model

Heterogeneity: $I^{2}=0 \%, T^{2}=0, p=0.48$

Random effects model

Heterogeneity: $I^{2}=0 \%, T^{2}=0, p=0.81$

Test for subgroup differences: $\mathrm{X}_{1}^{2}=2.27, \mathrm{df}=1 \quad(p=0.13)$

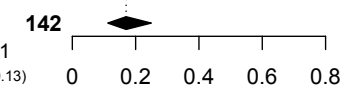

B

Proportion $\quad 95 \%-\mathrm{Cl}$ Weight

$\begin{array}{rr}0.08[0.01 ; 0.25] & 11.3 \% \\ 0.21[0.07 ; 0.42] & 24.2 \% \\ 0.00[0.00 ; 0.84] & 2.5 \% \\ 0.33[0.01 ; 0.91] & 4.1 \% \\ 0.00[0.00 ; 0.60] & 2.8 \% \\ 0.00[0.00 ; 0.52] & 2.8 \% \\ 0.50[0.01 ; 0.99] & 3.1 \% \\ 0.00[0.00 ; 0.60] & 2.8 \% \\ 0.00[0.00 ; 0.84] & 2.5 \% \\ 0.23[0.09 ; 0.44] & 28.2 \% \\ 0.25[0.01 ; 0.81] & 4.6 \% \\ 0.19[0.12 ; 0.28] & 88.9 \%\end{array}$

$0.00[0.00 ; 0.37] \quad 2.9 \%$

$0.00[0.00 ; 0.14] \quad 3.0 \%$

$0.14[0.00 ; 0.58] \quad 5.2 \%$

$0.07[0.02 ; 0.23] \quad 11.1 \%$

$0.17[0.11 ; 0.25] 100.0 \%$

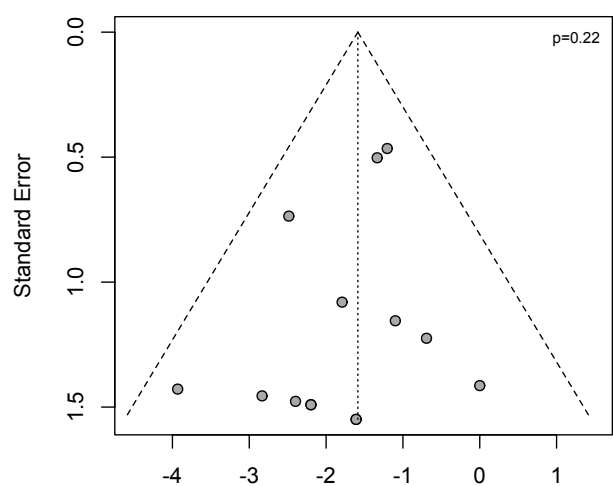

Recurrent Endocarditis

Figure 3 Mean recurrent endocarditis rates for patients who underwent valvectomy $v s$. tricuspid valve replacement. (A) Forest plot; (B) funnel plot.

reoperation for staged tricuspid valve replacement.

Medical management for tricuspid valve endocarditis remains the mainstay of therapy, with surgery reserved for cases of infective endocarditis complications, hemodynamic instability, failed medical therapy, persistent embolization, or large perivalvular vegetations (3). Optimal surgical treatment for infective tricuspid valve endocarditis in patients with IVDU remains a significant therapeutic challenge. Surgical options range from valve repair or replacement to the removal of the tricuspid valve leaflets and chordae tendinae without replacement (valvectomy) (3).

The three basic principles cited for the successful surgical treatment of infective endocarditis are (I) aggressive and extensive debridement of vegetations; (II) correction of defects that have developed; (III) whenever possible, to repair the valve with homologous or autologous pericardium 
A Study

Events Total

V2 = Valve_replacement Furat, Cevdet

Pomar, Jose L

Gottardi, Roman

Couetil, Jean-Paul A

Shrestha, Bishwo

Shetty, Nabha

Baraki, Hassina

Jiang, Sheng-li

Morokuma, Hiroyuki

Mahmod, Syed Usman Bin

Random effects model

Heterogeneity: $I^{2}=0 \%, T^{2}=0, p=0.97$

V2 = Valvectomy

Rouviex, E

Protos, Adam

Mahmod, Syed Usman Bin

Random effects model

Heterogeneity: $I^{2}=70 \%, T^{2}=2.0390, p=0.04$

Random effects model

Heterogeneity: $I^{2}=47 \%, T^{2}=1.0336, p=0.03$

Test for subgroup differences: $X_{1}^{2}=3.58, \mathrm{df}=1(p=0.06)$

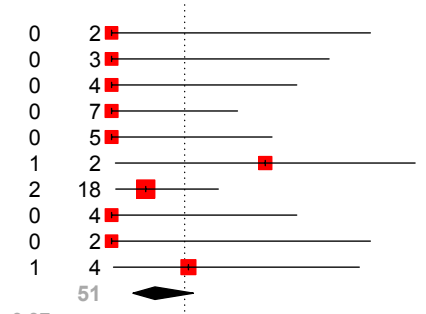

Proportion

95\%-Cl Weight

\begin{tabular}{|c|c|c|}
\hline 00 & {$[0.00 ; 0.84]$} & \\
\hline 00 & & \\
\hline & & \\
\hline & & \\
\hline 0 & & $6.1 \%$ \\
\hline 50 & {$[0.0$} & $6.4 \%$ \\
\hline 1 & {$[0.01 ; 0.3$} & $12.3 \%$ \\
\hline 0 & {$[0.00 ; 0$.} & $6.0 \%$ \\
\hline 0 & {$[0.00 ; 0$.} & $5.7 \%$ \\
\hline & {$[0.01 ; 0.8$} & \\
\hline & $=07 \cdot 0.27$ & $68.5^{\circ}$ \\
\hline
\end{tabular}

B

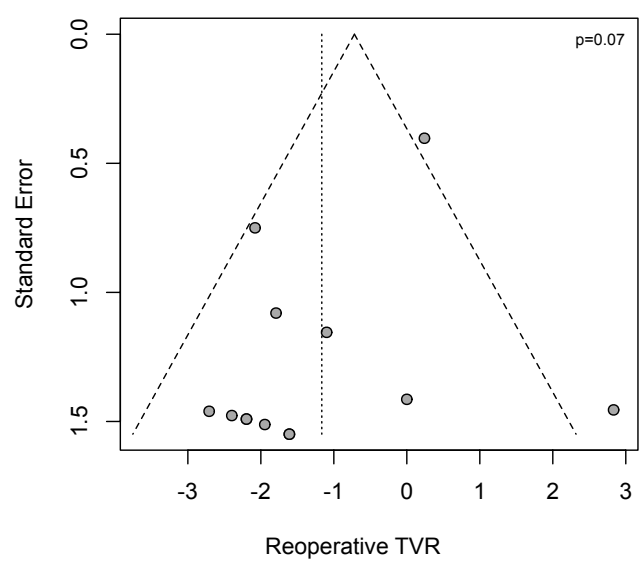

$0.24[0.12 ; 0.43] 100.0 \%$

Figure 4 Mean re-operation rates for those who underwent valvectomy $v$ s. tricuspid valve replacement. (A) Forest plot; (B) funnel plot.

\begin{tabular}{|c|c|c|c|c|c|c|c|c|c|c|}
\hline \multirow{2}{*}{$\begin{array}{l}\text { Time from } \\
\text { operation }\end{array}$} & \multicolumn{4}{|c|}{ Valve replacement } & \multicolumn{4}{|l|}{ Valvectomy } & \multicolumn{2}{|l|}{ Overall } \\
\hline & $\begin{array}{l}\text { Pooled value } \\
{[95 \% \mathrm{Cl}]}\end{array}$ & $\begin{array}{l}\text { No. of } \\
\text { studies }\end{array}$ & $\begin{array}{l}\text { No. of } \\
\text { patients }\end{array}$ & $\mathrm{I}^{2}(\%)$ & $\begin{array}{l}\text { Pooled value } \\
{[95 \% \mathrm{Cl}]}\end{array}$ & $\begin{array}{l}\text { No. of } \\
\text { studies }\end{array}$ & $\begin{array}{l}\text { No. of } \\
\text { patients }\end{array}$ & $I^{2}(\%)$ & $P$ value & $I^{2}(\%)$ \\
\hline 30 days & 88 [77-95] & 8 & $48 / 51$ & 0 & 96 [82-99] & 3 & $40 / 40$ & 0 & 0.23 & 0 \\
\hline 3 months & 89 [73-96] & 7 & $27 / 27$ & 0 & 96 [82-99] & 3 & $40 / 40$ & 0 & 0.30 & 0 \\
\hline 6 months & 89 [73-96] & 7 & $27 / 27$ & 0 & 97 [79-100] & 2 & $32 / 32$ & 0 & 0.30 & 0 \\
\hline 9 months & 89 [73-96] & 7 & $27 / 27$ & 0 & 97 [79-100] & 2 & $32 / 32$ & 0 & 0.30 & 0 \\
\hline 1 year & 89 [73-96] & 7 & $27 / 27$ & 0 & 46 [8-90] & 3 & $18 / 40$ & $72^{*}$ & 0.06 & 62 \\
\hline All & 86 [73-93] & 10 & $47 / 51$ & 0 & 44 [10-85] & 3 & $17 / 40$ & $70^{*}$ & 0.06 & 36 \\
\hline
\end{tabular}

to avoid implantation of artificial material (9).

As the majority of centers prioritize tricuspid valve repair, whereby valvectomy or valve replacement was only performed after attempts to repair were unsuccessful (10), we aimed to compare the outcomes of valvectomy versus valve replacement. Valvectomy without replacement results in massive tricuspid regurgitation and ventricularization of right atrial pressures as a result of a larger $\mathrm{V}$ wave $(11,12)$. The advantages of valvectomy include the limitation of foreign material, avoidance of the need for anticoagulation therapy and lower risk of heart block necessitating a permanent pacemaker (10). Significant right heart failure can develop in patients who undergo tricuspid valvectomy $(11,12)$. Valve replacement with either a biological or mechanical valve exposes the patient to valve-related complications, heart block requiring a pacemaker and risk of recurrent endocarditis $(11,12)$. We demonstrate that total tricuspid valvectomy without replacement can be a lifesaving measure in patients with IVDU and severe tricuspid lesions that can effectively eradicate the focus of infection with similar perioperative outcomes between the groups. Of note, at one year, freedom from reoperation was lower in the valvectomy group as compared to those who underwent replacement, however, this difference did not reach 
A Study

Events Total

V2 = Valve_replacement Xu, Zhiwei Gaca, Jeffery G Pomar, Jose L Gottardi, Roman Couetil, Jean-Paul A Shrestha, Bishwo Baraki, Hassina Jiang, Sheng-li Musci, Michele Morokuma, Hiroyuki Protos, Adam

Mahmod, Syed Usman Bin Random effects model Heterogeneity: $I^{2}=0 \%, T^{2}=0, p=0.78$

\section{$\mathrm{V} 2=$ Valvectomy}

Gaca, Jeffery G

Rouviex, E

Protos, Adam

Mahmod, Syed Usman Bin

Random effects model

Heterogeneity: $I^{2}=46 \%, T^{2}=0.4942, p=0.14$

Random effects model

Test for subgren: $I^{2}=11 \%, T^{2}=0.0627, p=0.33$

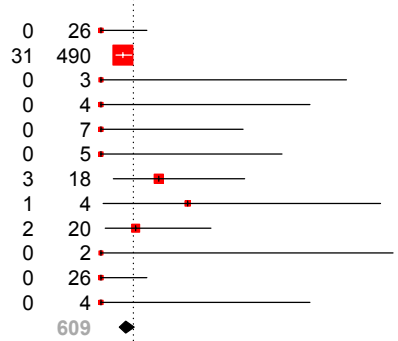

Proportion

$95 \%-\mathrm{Cl}$ Weight

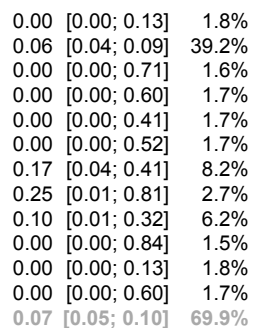

$0.12[0.05 ; 0.22] 18.6 \%$

$0.38[0.09 ; 0.76] \quad 6.4 \%$

$0.04[0.00 ; 0.20] \quad 3.4 \%$

$0.00[0.00 ; 0.41] \quad 1.7 \%$

$0.13[0.05 ; 0.30] \quad 30.1 \%$

$0.09[0.07 ; 0.13] 100.0 \%$
B

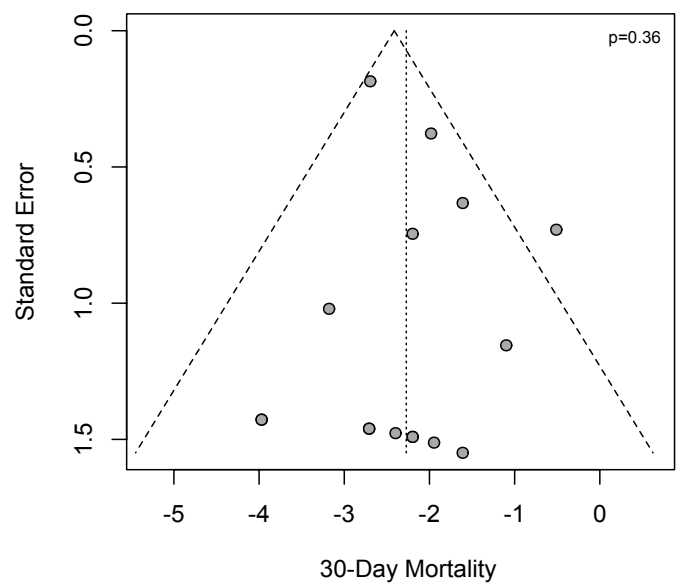

Figure 5 Mean 30-day postoperative mortality rates reported for patients who underwent valvectomy vs. tricuspid valve replacement. (A) Forest plot; (B) funnel plot.

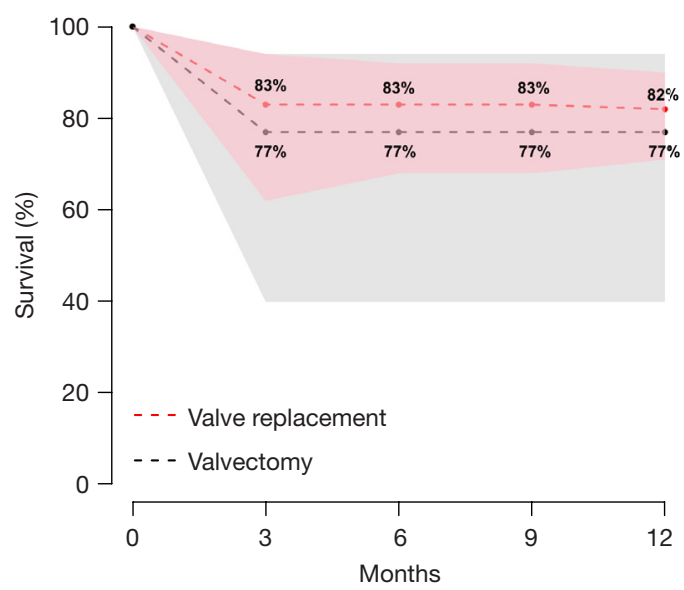

Figure 6 Overall survival as stratified by patients with intravenous drug use associated infective tricuspid valve endocarditis who underwent tricuspid valvectomy $v$ s. tricuspid valve replacement for the index procedure.

statistical significance. Lower freedom from reoperation would mean that either the tricuspid valve was replaced electively in those patients with valvectomy after completion of drug rehabilitation, or decompensation with heart failure between 9 and 12 months following valvectomy required tricuspid valve replacement.

In a single-center retrospective study comparing patients with IVDU-associated isolated tricuspid infective endocarditis, there were no differences in 30-day operative mortality, bleeding requiring reoperation, major stroke, prolonged ventilator time, intensive care unit and hospital length of stay between patients who underwent tricuspid valvectomy or replacement (13). Furthermore, Protos et al. (13) demonstrated that patients who underwent tricuspid valvectomy had significantly lower unplanned hospital readmission rates at 1 year, compared to those who underwent replacement. An important recent study of surgical outcomes for isolated IVDU-associated tricuspid valve infective endocarditis, utilizing The Society of Thoracic Surgeons (STS) adult cardiac surgery database, and using a contemporary cohort of patients demonstrates that valvectomy was an independent predictor of operative mortality (14).

Prior reports have demonstrated that long-term survival without a tricuspid valve is feasible (up to 22 years) in the context of normal pulmonary artery pressures and/ or pulmonary vascular resistance $(11,15)$. The lack of significant differences in perioperative outcomes, coupled with high prosthetic valve infection and readmission rates seen in patients treated with valve replacement at their initial operation, helps to establish tricuspid valvectomy as a useful bridge to staged valve replacement in this highrisk patient population. However, it is important to note 
that due to the lack of granularity in results, we were unable to evaluate for pre-operative left-sided heart function, pulmonary vascular resistance and pulmonary artery pressures which have been shown to predict the ability of the heart to withstand the absence of a tricuspid valve $(11,12)$. Furthermore, our study may be underpowered to detect significant difference.

Patients who undergo valvectomy were not at a disadvantage compared with those who underwent initial replacement. Tricuspid valvectomy can be a feasible option in patients with active ongoing IVDU, normal pulmonary pressure, normal biventricular heart function, high degree of valvular destruction and high risk of reoperation, recidivism and recurrence for infection (16). Valvectomy with valve replacement as a staged procedure can allow patients to self-select in terms of their ability to maintain adequate follow-up, undergo detoxification and drug rehabilitation, optimize their social and financial situation, and demonstrate abstinence from IVDU prior to tricuspid valve replacement. Furthermore, the complete absence of the tricuspid valve with valvectomy can prevent recurrence of infection.

The decision of choice and whether to offer surgical therapy for patients with IVDU-associated infective tricuspid valve endocarditis is a complex one-with social considerations (willingness to abstain from IVDU, compliance with antibiotic therapy stable income, stable housing, detoxification and rehabilitation), clinical considerations (size of vegetations, extension of abscess, comorbid HIV and hepatitis C infection) and ethical considerations (prolong life expectancy, risk of recurrence, healthcare expenditures and resource allocation) (15). IVDU is an independent predictor of death in patients with endocarditis and should be considered strongly in surgical recommendations for this complicated population $(1,13,17)$.

Prior literature suggests that addiction treatment for patients with infective endocarditis remains suboptimal (18). Every interaction with a patient with IVDU should be looked upon as an attempt to educate and encourage cessation of drug use and to undergo detoxification or rehabilitation. Improved addiction interventions, signed agreements for abstinence and a multidisciplinary heartteam approach (cardiac surgeons, intensivists, internalists, infectious disease specialists, psychiatrists, social workers, addiction team, ethicists, etc.) in the decision-making process are imperative in both the medical and surgical management of IVDU-associated infective endocarditis, and cannot be understated. Optimal treatment of addiction combines psychosocial and financial supports, medicationassisted treatment and harm-reduction strategies including overdose education and naloxone distribution (18).

In addition to surgical options of valvectomy or replacement, novel methods of percutaneous nonsurgical debridement of tricuspid valve vegetations are available: promising results from use of the AngioVac (AngioDynamics, Latham, NY) system with suction filtration on veno-venous extracorporeal bypass have been reported (19). Further evaluation is warranted regarding this potentially paradigmshifting alternative to open heart surgery in this high-risk population.

\section{Limitations}

The present meta-analysis has several key limitations and must be interpreted with care. Due to sparse data and lack in granularity of the studies available, we were unable to stratify postoperative outcomes of valvectomy versus replacement for patients with IVDU infective tricuspid endocarditis based on septic pulmonary embolism, pulmonary hypertension, pulmonary vascular resistance and preoperative right and left heart function. Regional differences exist in patient selection, surgical techniques, preoperative, perioperative and postoperative management, medications, addictions counseling, rehabilitation and follow-up. Outcomes in patients' post-surgery as stratified by compliance, abstinence and continued IVDU was lacking in the included studies, and as such, the effect of this on outcomes was not assessed. It is possible that our study is underpowered to detect significant differences between groups. These limitations restrict the broader applicability of the results presented in this study. We acknowledge that this heterogeneity in study population is a fundamental limitation that cannot be addressed due to inability to extract sufficient detail from the pooled data. Publication bias as well as the small number of patients limits the statistical power of the analysis.

\section{Conclusions}

Tricuspid valvectomy is an acceptable initial therapy for infective tricuspid valve endocarditis in patients with IVDU as a bridge to identify those who will self-select themselves as candidates for staged valve replacement. There is a need for high-quality prospective data on the surgical management of IVDU-associated isolated infective 
tricuspid endocarditis.

\section{Acknowledgments}

None.

\section{Footnote}

Conflicts of Interest: The authors have no conflicts of interest to declare.

\section{References}

1. Shrestha NK, Jue J, Hussain ST, et al. Injection Drug Use and Outcomes After Surgical Intervention for Infective Endocarditis. Ann Thorac Surg 2015;100:875-82.

2. Baddour LM, Wilson WR, Bayer AS, et al. Infective endocarditis: diagnosis, antimicrobial therapy, and management of complications: a statement for healthcare professionals from the Committee on Rheumatic Fever, Endocarditis, and Kawasaki Disease, Council on Cardiovascular Disease in the Young, and the Councils on Clinical Cardiology, Stroke, and Cardiovascular Surgery and Anesthesia, American Heart Association: endorsed by the Infectious Diseases Society of America. Circulation 2005;111:e394-434.

3. Nishimura RA, Otto CM, Bonow RO, et al. 2017 AHA/ACC Focused Update of the 2014 AHA/ACC Guideline for the Management of Patients With Valvular Heart Disease: A Report of the American College of Cardiology/American Heart Association Task Force on Clinical Practice Guidelines. J Am Coll Cardiol 2017;70:252-89.

4. AATS Surgical Treatment of Infective Endocarditis Consensus Guidelines Writing Committee Chairs, Pettersson GB, Coselli JS, et al. 2016 The American Association for Thoracic Surgery (AATS) consensus guidelines: Surgical treatment of infective endocarditis: Executive summary. J Thorac Cardiovasc Surg 2017;153:1241-58.e29.

5. Baddour LM, Wilson WR, Bayer AS, et al. Infective Endocarditis in Adults: Diagnosis, Antimicrobial Therapy, and Management of Complications: A Scientific Statement for Healthcare Professionals From the American Heart Association. Circulation 2015;132:1435-86.

6. Hussain ST, Witten J, Shrestha NK, et al. Tricuspid valve endocarditis. Ann Cardiothorac Surg 2017;6:255-61.

7. Margulis AV, Pladevall M, Riera-Guardia N, et al. Quality assessment of observational studies in a drug-safety systematic review, comparison of two tools: the NewcastleOttawa Scale and the RTI item bank. Clin Epidemiol 2014;6:359-68.

8. Wang X, Tang K, Chen L, et al. Association between sepsis and retinopathy of prematurity: a systematic review and meta-analysis. BMJ Open 2019;9:e025440.

9. Musci M, Siniawski H, Pasic M, et al. Surgical treatment of right-sided active infective endocarditis with or without involvement of the left heart: 20-year single center experience. Eur J Cardiothorac Surg 2007;32:118-25.

10. Yanagawa B, Elbatarny M, Verma S, et al. Surgical Management of Tricuspid Valve Infective Endocarditis: A Systematic Review and Meta-Analysis. Ann Thorac Surg 2018;106:708-14.

11. Arbulu A, Holmes RJ, Asfaw I. Surgical treatment of intractable right-sided infective endocarditis in drug addicts: 25 years experience. J Heart Valve Dis 1993;2:12937; discussion 138-9.

12. Robin E, Thomas NW, Arbulu A, et al. Hemodynamic consequences of total removal of the tricuspid valve without prosthetic replacement. Am J Cardiol 1975;35:481-6.

13. Protos AN, Trivedi JR, Whited WM, et al. Valvectomy Versus Replacement for the Surgical Treatment of Tricuspid Endocarditis. Ann Thorac Surg 2018;106:664-9.

14. Slaughter MS, Badhwar V, Ising M, et al. Optimum Surgical Treatment for Tricuspid Valve Infective Endocarditis: Analysis of the STS National Database. AATS 2019 Conf Abstr 2019. Available online: https:// www.aats.org/aatsimis/AATSWeb/Association/Meetings/ Annual_Meeting/99th_Annual_Meeting/AATS_99th_ Annual_Meeting_Abstracts/2019-A-1303-AATS-283. aspx

15. Gansera LS, Eszlari E, Deutsch O, et al. High-Risk Cardiac Surgery in Patients with Intravenous Drug Abuse and/or Active Hepatitis C or HIV Infection: An Ethical Discussion of Six Cases. Thorac Cardiovasc Surg 2016;64:2-5.

16. Munro AI, Jamieson WR, Tyers GF, et al. Tricuspid valve replacement: porcine bioprostheses and mechanical prostheses. Ann Thorac Surg 1995;60:S470-3; discussion S473-4.

17. Baraki H, Saito S, Al Ahmad A, et al. Surgical treatment for isolated tricuspid valve endocarditis- long-term followup at a single institution. Circ J 2013;77:2032-7.

18. Rosenthal ES, Karchmer AW, Theisen-Toupal J, et al. Suboptimal Addiction Interventions for Patients 
Hospitalized with Injection Drug Use-Associated Infective Endocarditis. Am J Med 2016;129:481-5.

19. George B, Voelkel A, Kotter J, et al. A novel approach to percutaneous removal of large tricuspid valve vegetations

Cite this article as: Luc JGY, Choi JH, Kodia K, Weber MP, Horan DP, Maynes EJ, Carlson LA, Massey HT, Entwistle JW, Morris RJ, Tchantchaleishvili V. Valvectomy versus replacement for the surgical treatment of infective tricuspid valve endocarditis: a systematic review and meta-analysis. Ann Cardiothorac Surg 2019;8(6):610-620. doi: 10.21037/ acs.2019.11.06 using suction filtration and veno-venous bypass: A single center experience. Catheter Cardiovasc Interv 2017;90:1009-15. 
Table S1 Characteristics and quality assessment of studies included.

\begin{tabular}{|c|c|c|c|c|c|c|}
\hline Title & Authors & $\begin{array}{l}\text { Year } \\
\text { published }\end{array}$ & Journal & Study years & Study type & $\begin{array}{l}\text { Newcastle- } \\
\text { Ottawa Scale }\end{array}$ \\
\hline $\begin{array}{l}\text { Valvectomy versus replacement for the surgical } \\
\text { treatment of tricuspid endocarditis (13) }\end{array}$ & $\begin{array}{l}\text { Protos } \\
\text { et al. }\end{array}$ & 2018 & Ann Thorac Surg & 2012-2016 & Retrospective & 5 \\
\hline $\begin{array}{l}\text { Isolated Tricuspid Valvectomy: A Series of } \\
\text { cases with Intravenous Drug Abuse Associated } \\
\text { Tricuspid Valve Endocarditis (20) }\end{array}$ & $\begin{array}{l}\text { Bin } \\
\text { Mahmood } \\
\text { et al. }\end{array}$ & 2018 & $\begin{array}{l}\text { Thorac } \\
\text { Cardiovasc Surg }\end{array}$ & 2009-2017 & Retrospective & 6 \\
\hline $\begin{array}{l}\text { Surgical and Medical Management of Isolated } \\
\text { Tricuspid Valve Infective Endocarditis in } \\
\text { Intravenous Drug Users (22) }\end{array}$ & $\begin{array}{l}\text { Shetty } \\
\text { et al. }\end{array}$ & 2016 & J Card Surg & 2008-2011 & Retrospective & 8 \\
\hline $\begin{array}{l}\text { Isolated tricuspid valve infective endocarditis in } \\
\text { young drug abusers (24) }\end{array}$ & Furat et al. & 2014 & $\begin{array}{l}\text { Ther Adv } \\
\text { Cardiovasc Dis }\end{array}$ & 2014 & Case Series & 5 \\
\hline $\begin{array}{l}\text { Current Outcomes for Tricuspid Valve Infective } \\
\text { Endocarditis Surgery in North America (25) }\end{array}$ & Gaca et al. & 2013 & Ann Thorac Surg & 2002-2009 & Retrospective & 5 \\
\hline $\begin{array}{l}\text { Surgical treatment for isolated tricuspid valve } \\
\text { endocarditis (17) }\end{array}$ & $\begin{array}{l}\text { Baraki } \\
\text { et al. }\end{array}$ & 2013 & Circ J & 1996-2012 & Retrospective & 6 \\
\hline $\begin{array}{l}\text { Surgical treatment of isolated right-sided } \\
\text { infective endocarditis (26) }\end{array}$ & Jiang et al. & 2011 & Tex Heart Inst J & 2000-2010 & Retrospective & 6 \\
\hline $\begin{array}{l}\text { Partial Replacement of Tricuspid Valve Using } \\
\text { Cryopreserved Homograft (27) }\end{array}$ & $\begin{array}{l}\text { Shrestha } \\
\text { et al. }\end{array}$ & 2010 & Ann Thorac Surg & $1997-2008$ & Retrospective & 6 \\
\hline $\begin{array}{l}\text { Surgical treatment of right-sided active infective } \\
\text { endocarditis with or without involvement of the } \\
\text { left heart: } 20 \text {-year single center experience (9) }\end{array}$ & $\begin{array}{l}\text { Musci } \\
\text { et al. }\end{array}$ & 2007 & $\begin{array}{l}\text { Eur J } \\
\text { Cardiothorac } \\
\text { Surg }\end{array}$ & 1986-2006 & Retrospective & 8 \\
\hline $\begin{array}{l}\text { Partial replacement of the tricuspid valve by } \\
\text { mitral homografts in acute endocarditis ( } 30)\end{array}$ & $\begin{array}{l}\text { Couetil } \\
\text { et al. }\end{array}$ & 2002 & Ann Thorac Surg & 2002 & Retrospective & 6 \\
\hline $\begin{array}{l}\text { Management of persistent tricuspid endocarditis } \\
\text { with transplantation of cryopreserved mitral } \\
\text { homografts (31) }\end{array}$ & $\begin{array}{l}\text { Pomar } \\
\text { et al. }\end{array}$ & 1994 & $\begin{array}{l}J \text { Thorac } \\
\text { Cardiovasc Surg }\end{array}$ & 1993-1994 & Case series & 6 \\
\hline Tricuspid infective endocarditis: 56 cases (32) & $\begin{array}{l}\text { Rouveix } \\
\text { et al. }\end{array}$ & 1984 & Eur Heart $J$ & 1968-1983 & Retrospective & 8 \\
\hline
\end{tabular}




\section{References}

20. Bin Mahmood SU, Nguemeni Tiako MJ, Mori M, et al. Isolated Tricuspid Valvectomy: A Series of cases with Intravenous Drug Abuse Associated Tricuspid Valve Endocarditis. Thorac Cardiovasc Surg 2018. [Epub ahead of print].

21. Xu Z, Shi Q, Mei J, et al. Bioprosthetic tricuspid valve replacement for tricuspid valve endocarditis secondary to infected endocardial pacemaker leads. J Card Surg 2017;32:5-8.

22. Shetty N, Nagpal D, Koivu S, et al. Surgical and Medical Management of Isolated Tricuspid Valve Infective Endocarditis in Intravenous Drug Users. J Card Surg 2016;31:83-8.

23. Dawood MY, Cheema FH, Ghoreishi M, et al. Contemporary outcomes of operations for tricuspid valve infective endocarditis. Ann Thorac Surg 2015;99:539-46.

24. Furat $\mathrm{C}$, Ilhan $\mathrm{G}$, Bayar E, et al. Isolated tricuspid valve infective endocarditis in young drug abusers. Ther Adv Cardiovasc Dis 2014;8:119-22.

25. Gaca JG, Sheng S, Daneshmand M, et al. Current Outcomes for Tricuspid Valve Infective Endocarditis
Surgery in North America. Ann Thorac Surg 2013;96:1374-81.

26. Jiang SL, Li BJ, Zhang T, et al. Surgical treatment of isolated right-sided infective endocarditis. Tex Heart Inst J 2011;38:639-42.

27. Shrestha BMS, Fukushima S, Vrtik M, et al. Partial Replacement of Tricuspid Valve Using Cryopreserved Homograft. Ann Thorac Surg 2010;89:1187-94.

28. Morokuma H, Minato N, Kamohara K, et al. Three surgical cases of isolated tricuspid valve infective endocarditis. Ann Thorac Cardiovasc Surg 2010;16:134-8.

29. Gottardi R, Bialy J, Devyatko E, et al. Midterm Follow-Up of Tricuspid Valve Reconstruction Due to Active Infective Endocarditis. Ann Thorac Surg 2007;84:1943-8.

30. Couetil J-PA, Argyriadis PG, Shafy A, et al. Partial replacement of the tricuspid valve by mitral homografts in acute endocarditis. Ann Thorac Surg 2002;73:1808-12.

31. Pomar JL, Mestres CA, Pare JC, et al. Management of persistent tricuspid endocarditis with transplantation of cryopreserved mitral homografts. J Thorac Cardiovasc Surg 1994;107:1460-3.

32. Rouveix E, Witchitz S, Bouvet E, et al. Tricuspid infective endocarditis: 56 cases. Eur Heart J 1984;5 Suppl C:111-5. 